\title{
Chronic diabetic complications in patients with MODY3 diabetes
}

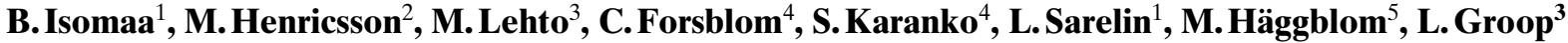 \\ ${ }^{1}$ Jakobstad Hospital, Jakobstad, Finland \\ ${ }^{2}$ Helsingborg Hospital, Helsingborg, Sweden \\ ${ }^{3}$ Department of Endocrinology, Lund University, Malmö, Sweden \\ ${ }^{4}$ Department of Medicine, Helsinki University Hospital, Helsinki, Finland \\ ${ }^{5}$ Närpes Health Center, Närpes, Finland
}

Summary MODY3 diabetes, which is caused by a mutation in the hepatocyte nuclear factor- $1 \alpha$ gene (HNF-1 $\alpha$ ) on chromosome 12, represents a relatively common monogenic form of diabetes in Finland. Age at onset of the disease can vary from 10 to 60 years, but little is known about the natural course of the disease, particularly the development of diabetes-related chronic complications. The availability of genetic markers now allows description of the clinical course of the disease. In order to examine the prevalence of chronic diabetic complications in MODY3, we examined 57 carriers with HNF-1 $\alpha$ mutations for the presence of micro- and macrovascular complications. Thirty-four percent of the MODY patients had mild and $13 \%$ had severe non-proliferative or proliferative retinopathy; this figure did not differ from the figures in insulin-dependent diabetes mellitus (IDDM) and non-insulin-dependent diabetes mellitus (NIDDM) patients matched for duration and glycaemic control but not for age. Neither did the prevalence of microalbuminuria differ between
MODY3 and IDDM or NIDDM patients (19 vs 24 and $23 \%$ ). Neuropathy was observed with the same frequency as previously reported in IDDM. Hypertension was less frequent in MODY3 and IDDM than in NIDDM (24.5 and 19 vs $53.7 \% ; p<0.001)$. Coronary heart disease was more common in MODY 3 than in IDDM (16 vs $4.5 \% ; p<0.02)$ but less common than in the older NIDDM patients $(33.3 \% ; p<0.02)$. In a multiple logistic regression analysis, poor glycaemic control was an independent risk factor for retinopathy ( $p=0.03)$, microalbuminuria $(p<0.04)$ and neuropathy $(p=0.03)$. In conclusion, microangiopathic complications are observed with the same frequency in patients with MODY3 diabetes as in IDDM and NIDDM and are strongly related to poor glycaemic control. [Diabetologia (1998) 41: 467-473]

Keywords MODY3, NIDDM, complications, retinopathy, neuropathy, microalbuminuria, cardiovascular disease, hypertension.
Non-insulin-dependent diabetes mellitus (NIDDM) is a heterogeneous disorder with a complex pattern of inheritance; it usually becomes manifest after

Received: 24 June 1997 and in revised form: 3 December 1997

Corresponding author: Professor L. C. Groop, Department of Endocrinology, University Hospital Malmö, University of Lund, S-205 02 Malmö, Sweden

Abbreviations: CI, Confidence interval; DR, diabetic retinopathy; NIDDM, non-insulin-dependent diabetes mellitus; NPDR, non-proliferative diabetic retinopathy; MODY, maturity-onset diabetes in the young; PDR, proliferative retinopathy; RR, relative risk; BMI, body mass index; WHR, waist-hip ratio.
40 years of age and the course of the disease is generally determined by the severity of concomitant macrovascular complications. In contrast to adult-onset NIDDM, maturity-onset diabetes of the young (MODY) shows a clear dominant inheritance, early onset and high penetrance $[1,2]$. MODY is thought to account for less than $5 \%$ of all cases with NIDDM, but its true prevalence is unknown. MODY itself is a heterogeneous disease. Three distinct forms of MODY have been described, the rare MODY1 which is caused by mutations in a transcription factor gene, hepatocyte nuclear factor- $4 \alpha(\mathrm{HNF}-4 \alpha)$ on chromosome 20 [3], the mild MODY2, which is caused by several mutations in the glucokinase gene 
Table 1. Comparison between MODY3 IDDM and NIDDM patients included in the comparison of nephropathy

\begin{tabular}{|c|c|c|c|}
\hline Number (male/female) & $\begin{array}{l}\text { MODY } \\
57(29 / 28)\end{array}$ & $\begin{array}{l}\text { IDDM } \\
111(67 / 44)\end{array}$ & $\begin{array}{l}\text { NIDDM } \\
159(63 / 96)\end{array}$ \\
\hline Age (years) & $43.7 \pm 15.7$ & $38.6 \pm 12.5$ & $69.4 \pm 11.6^{b}$ \\
\hline Duration (years) & $17.0 \pm 12.8$ & $23.1 \pm 12.0$ & $22.9 \pm 6.1$ \\
\hline BMI $\left(\mathrm{kg} / \mathrm{m}^{2}\right)$ & $24.6 \pm 4.5$ & $25.1 \pm 3.2$ & $27.5 \pm 4.9^{\mathrm{b}}$ \\
\hline Waist hip ratio & $0.89 \pm 0.08$ & N.D. & $0.93 \pm 0.08$ \\
\hline $\mathrm{HbA}_{1 \mathrm{c}}(\%)$ & $7.3 \pm 1.6$ & $7.7 \pm 1.5$ & $7.6 \pm 1.9$ \\
\hline Cholesterol (mmol/l) & $5.3 \pm 1.0$ & $5.0 \pm 0.9$ & $5.9 \pm 1.3^{\mathrm{a}}$ \\
\hline HDL cholesterol (mmol/l) & $1.40 \pm 0.21$ & $1.45 \pm 0.40$ & $1.25 \pm 0.40^{\mathrm{a}}$ \\
\hline Triglycerides $(\mathrm{mmol} / \mathrm{l})$ & $1.53 \pm 0.82$ & $1.01 \pm 0.50$ & $1.86 \pm 1.04^{\mathrm{a}}$ \\
\hline AER > $20 \mu \mathrm{g} / \mathrm{min}(\%)$ & 19 & 24 & 23 \\
\hline Hypertension (\%) & 24.5 & 19.0 & $53.7^{\mathrm{b}}$ \\
\hline Coronary heart disease (\%) & $16^{\mathrm{c}}$ & 4.5 & 33.3 \\
\hline Clinical neuropathy $(\%)$ & $29 \%$ & $13 \%{ }^{b}$ & N.D. \\
\hline Vibration threshold (\%) & $40 \%$ & $41 \%$ & N.D. \\
\hline \multicolumn{4}{|l|}{ Diabetes therapy } \\
\hline Diet alone $(\%)$ & 33 & 0 & 60.3 \\
\hline Oral agents (\%) & 30 & 0 & 31.5 \\
\hline Insulin (\%) & 37 & 100 & 8.2 \\
\hline
\end{tabular}

Values are mean $\pm \mathrm{SD} ;{ }^{\mathrm{a}} p<0.01,{ }^{\mathrm{b}} p<0.001$ vs MODY or IDDM; ${ }^{\mathrm{c}} p<0.02, p<0.001$ vs IDDM. Abnormal vibration threshold measured by Biothesiometer. N.D., not determined using standardised methodology

on chromosome 7 [4] and the relatively common MODY3, which is caused by mutations in another transcription factor gene, HNF-1 $\alpha$ on chromosome 12 [5]. Little phenotypic information has been available on the different forms of MODY $[6,7]$ particularly on the prevalence of diabetic complications. There is some evidence that MODY2 shows less complications than the other subtypes [6,7], but it is not known whether patients with MODY1 and 3 develop micro- and macroangiopathy in the same way as patients with NIDDM and insulin-dependent diabetes mellitus (IDDM). The identification of the underlying genetic defects now allows diagnosis of the MODY subtypes in an individual patient, and therefore the study of the clinical course of the disease. To address this question, we studied the prevalence of micro- and macroangiopathy in patients with MODY3 from five large Finnish families and contrasted the findings with those in patients with IDDM and NIDDM.

\section{Materials and methods}

Sixty-three patients with MODY3 mutations were identified from five large Finnish families (family A 9 patients, family B 8 patients, family C 16 patients, family D 26 patients and family $\mathrm{H} 4$ patients) [8]. Of the patients, 57 fulfilled the World Health Organisation (WHO) criteria for diabetes while 6 had impaired glucose tolerance. Only the MODY3 patients with manifest diabetes were examined for the presence of diabetic complications. Forty-nine patients from four families shared the same insertion mutation of exon 4 in the HNF-1 $\alpha$ gene whereas eight patients from the fifth family had a missense mutation in the HNF-1 $\alpha$ gene [9]. The clinical characteristics of the MODY3 patients and groups of IDDM $(n=111)$ and NIDDM $(n=159)$ patients matched for duration of diabetes and glycaemic control are shown in Table 1. The IDDM patients represented consecutive patients from the outpatient clinic at the Jakobstad hospital (Jakobstad, Finland), while the NIDDM control group was selected from the Botnia study [10].

Measurements of body mass index (BMI), waist-hip-ratio (WHR), $\mathrm{HbA}_{1 \mathrm{c}}$, total cholesterol, HDL cholesterol, and triglyceride concentrations and albumin excretion rate in urine were available from all subjects. All available MODY3 subjects $(n=38)$ underwent a clinical examination aiming at the detection of neuropathy, retinopathy, microalbuminuria and macrovascular disease.

Neuropathy: The diagnosis of distal sensory neuropathy was based upon a clinical examination, measurement of vibration threshold by a biothesiometer and a symptom score [11]. The clinical examination consisted of measurements of 1) achilles tendon reflexes with a grading from $0-2$ for each side $($ normal $=0)$, present with reinforcement $=1$ and absent $=2$ ) giving a maximum score of 4 if both sides were affected, 2) vibration sensation measured by a $128 \mathrm{~Hz}$ vibration fork at the hallux with a grading from 0 to 1 for each side (normal $=0$, absent $=1$ ) giving a maximum score of 2 if both sides were affected, 3) sensory perception measured by a $10 \mathrm{~g}$ Semmes-Weinstein monofilament at three sites on each foot with a grading from 0 to 2 for each side (normal $=0$, absent on one site $=1$, absent on 2 or 3 sites $=2$ ) giving a maximum score of 4 if both sites were maximally affected. A score of 3-5 was regarded as mild neuropathy, a score of $6-8$ as moderate neuropathy and a score of 9-10 as severe distal neuropathy. For the subjective symptom score the patients answered a questionnaire [11]. A symptom score of 3-4 indicated mild symptoms, a score of 5-6 moderate symptoms while a score of 7-9 indicated severe symptomatic sensory neuropathy. For the multiple logistic regression analysis distal sensory polyneuropathy was considered present if the patient had moderate signs (score 6-8) with or without symptoms or mild signs (score 3-5) with moderate symptoms (score 5-6).

Neuropathy was also assessed by measuring the vibration perception threshold at ankle and hallux with a biothesiometer (Biomedical Instruments, Ohio, USA). As control, vibration perception threshold was also measured in 58 persons with normal glucose tolerance and without clinical signs or symptoms of neuropathy. An age-correlated threshold was calculated and a value exceeding 2 SD above the mean was considered 
Table 2. Comparison between MODY3 IDDM and NIDDM patients participating in the ophthalmologic examination

\begin{tabular}{lccc}
\hline Number (male/female) & MODY3 & IDDM & NIDDM \\
& $38(19 / 19)$ & $38(20 / 18)$ & $38(25 / 13)$ \\
\hline Age (years) & $46.3 \pm 17.4$ & $33.7 \pm 12.6$ & $58.9 \pm 12.4$ \\
Duration of diabetes (years) & $16.4 \pm 12.7$ & $16.1 \pm 12.9$ & $15.5 \pm 12.5$ \\
HbA, $(\%)$ & $6.8 \pm 1.2$ & $6.9 \pm 1.3$ & $7.1 \pm 1.3$ \\
Treatment & & & 29 \\
$\quad$ Diet alone (\%) & 34 & 0 & 16 \\
Oral agents (\%) & 29 & 100 & 55 \\
$\quad$ Insulin (\%) & 37 & 47 & 55 \\
Retinopathy & & 37 & 21 \\
No DR (\%) & 53 & 0 & 5 \\
Mild DR (\%) & 34 & 16 & 18 \\
Moderate DR (\%) & 0 & 3 & 8 \\
Severe NPDR or PDR (\%) & 13 & 8 & \\
Maculopathy & 8 & & \\
\hline
\end{tabular}

Values are mean \pm SD

pathological. There was a strong positive correlation between the biothesiometer measurements and the clinical neuropathy score in the MODY3 patients $(r=0.82 ; p<0.0001, n=36)$, while there was a weaker correlation between the biothesiometer measurements and the subjective symptom score $(r=$ $0.49, p=0.003, n=36$ ).

Microalbuminuria: Microalbuminuria was considered present if the albumin excretion rate (AER) in overnight urine exceeded $15 \mu \mathrm{g} / \mathrm{min}$ [12].

Retinopathy: The diagnosis of retinopathy was based upon fundus photography. Colour fundus photographs were obtained after pharmacological mydriasis at an angle of 45 degrees with a Topcon TRC NW 5 fundus camera (Tokyo Optical Co. Ltd., Tokyo, Japan). The photographs covered fields 1-3 of the seven standard fields, with stereo pairs of the macula (field 2). The alternative classification of the Wisconsin Epidemiological Study of Diabetic Retinopathy was used to obtain the retinopathy level $[13,14]$.

Briefly, level 10 represents no retinopathy, levels 21 through 51 non-proliferative diabetic retinopathy of increasing severity, and level $60+$ all forms of proliferative diabetic retinopathy, with and without laser treatment. The patient's retinopathy level was derived by giving the eye with the higher level a greater weight. This scheme provides an 11-step scale: 10,21/ $10,21 / 21,31 /<31,31 / 31,41 /<41,41 / 41,51 /<51,51 / 51,60+/$ $<60+, 60+/ 60+$. Thereafter, we divided the retinopathy levels into 4 groups; no diabetic retinopathy (DR) (level 10), mild retinopathy (levels 21/10-31/31), moderate retinopathy (levels $41 /<41-51 /<51$ ), and severe NPDR (level 51/51) or PDR (level $60+/ 60+$ ).

Macular oedema was defined as presence of hard exudates and/or retinal thickening within one disc diameter of the centre of the macula and considered as present if at least one eye was affected.

Photographs were viewed against light boards using Donaldson's stereo viewer $(5 \times$ magnification $)$ and assessed by an independent grader (E.S.), with extensive previous experience using this classification. The grader was masked according to the patients clinical data. Two blind MODY3 patients were excluded from photography because of media opacities. Their retinopathy was graded as PDR according to information from patient records.

For the ophthalmologic comparison two separate diabetic control groups were created comprising IDDM $(n=38)$, and NIDDM $(n=38)$ patients from Helsingborg, Sweden. The patients were matched for duration and glycaemic control but not for age (Table 2).
Cardiovascular disease: A history of myocardial infarction or stroke was based upon information from hospital records. Also, a diagnosis of coronary heart disease was based upon hospital records or use of nitroglycerine and pathological findings in the resting ECG. The ECGs were coded by one of us (B. I.) using the Minnesota codes 1.1-2, 4.1-4, 5.5-3 and 7.1 [15].

Peripheral vascular disease: The diagnosis of peripheral vascular disease was based upon measurement of an ankle-brachial index (ABI). Ankle systolic pressure was measured after 5 min of rest in supine position using a Doppler device (Multi-Doplex II, Huntleigh Technology, Cardiff, UK). A standard blood pressure cuff was placed around the right ankle and the blood flow in arteria tibialis posterior was registered. Two measurements were done, followed by an auscultatory measurement of the systolic pressure in the right arm. An ABI-index less than 0.9 was considered diagnostic of peripheral vascular disease.

\section{Measurements and assays}

Waist hip ratio (WHR): Waist circumference was measured with a soft tape on standing subjects midway between the lowest rib and the iliac crest. Hip circumference was measured over the widest part of the gluteal region, and waist-to-hip ratio (WHR) as a measure of central obesity was calculated.

Blood pressure: Three blood pressure recordings were obtained from the right arm of a sitting person after $30 \mathrm{~min}$ of rest at 5-min intervals, and their mean value was calculated. Hypertension was considered present if the patient used antihypertensive drugs or had a blood pressure over 160/ $95 \mathrm{~mm} \mathrm{Hg}$.

$\mathrm{HbA}_{1 \mathrm{c}}$ : was determined with a high pressure liquid chromatography method (Kyoto Daichii, Kyoto, Japan) (reference value $<5.5 \%$ ). In the diabetic control group for retinopathy screening $\mathrm{HbA}_{1 \mathrm{c}}$ was measured by ion exchange chromatography (MonoS - HPLC, Pharmacia, Uppsala, Sweden) [16] (reference value $<5.5 \%)$. The two methods correlated strongly $(r=0.95 ; p<0.001 ; n=57)$. For the comparison of the MODY3 patients and the age-matched diabetic patients, the MonoS values $(x)$ were converted to standard $\mathrm{HbA}_{1 \mathrm{c}}$ values (y) by the following equation: $y=0.95 x+0.69$.

To determine how representative a single $\mathrm{HbA}_{1 \mathrm{c}}$ value was of glycaemic control during the last 5 years, we correlated the last $\mathrm{HbA}_{1 \mathrm{c}}$ value with sequential measurements (average 
Table 3. Multiple regression analysis with age-adjusted elevated vibration threshold ${ }^{\text {a }}$ as dependent variable

\begin{tabular}{|c|c|c|c|}
\hline Variable & Risk ratio & $95 \%$ Confidence interval & $p$ value \\
\hline Age (years) & 1.13 & $0.99-1.29$ & 0.08 \\
\hline Duration of diabetes (years) & 1.03 & $0.93-1.15$ & 0.57 \\
\hline Hypertension & 2.9 & $0.44-19.5$ & 0.27 \\
\hline Male sex & 6.7 & $1.01-40.3$ & 0.04 \\
\hline $\mathrm{HbA}_{1 \mathrm{c}}(\%)$ & 5.0 & $1.15-22.1$ & 0.03 \\
\hline \multicolumn{4}{|c|}{ Multiple logistic regression analysis with clinical neuropathy as dependent variable } \\
\hline Variable & Risk ratio & $95 \%$ Confidence interval & $p$ value \\
\hline Age (years) & 1.02 & $0.97-1.12$ & 0.7 \\
\hline Duration of diabetes (years) & 1.14 & $1.00-1.30$ & 0.04 \\
\hline $\mathrm{HbA}_{1 \mathrm{c}}(\%)$ & 1.2 & $0.3-4.9$ & 0.8 \\
\hline Hypertension & 4.2 & $0.8-21.6$ & 0.08 \\
\hline Male sex & 1.5 & $0.4-5.4$ & 0.5 \\
\hline
\end{tabular}

Complete data on neuropathy was available from 36 MODY patients; 11 of whom were considered to have symptoms or

Table 4. Multiple regression analysis with microalbuminuria defined as AER over $15 \mu \mathrm{g} / \mathrm{min}$ as dependent variable

\begin{tabular}{llll}
\hline Dependent variable & Risk ratio & $\begin{array}{l}\text { 95\% Confidence } \\
\text { interval }\end{array}$ & $p$ value \\
\hline $\begin{array}{l}\text { Age (years) } \\
\text { Duration of diabetes }\end{array}$ & 1.0 & $0.94-1.06$ & 0.6 \\
(years) & 0.93 & $0.84-1.04$ & 0.19 \\
HbA $_{1 \mathrm{c}}(\%)$ & 2.30 & $1.05-5.0$ & 0.04 \\
Hypertension $_{\text {Male sex }}$ & 0.81 & $0.26-2.4$ & 0.70 \\
Clinical neuropathy & 1.2 & $0.54-2.71$ & 0.63 \\
\hline
\end{tabular}

Complete data for the independent variables was available from 36 patients; 12 of whom had AER over $15 \mu \mathrm{g} / \mathrm{min}$

7.5 measurements) in 23 subjects. The most recent measurement of $\mathrm{HbA}_{1 \mathrm{c}}$ correlated strongly with the values measured during the past 5 years $(r=0.91 ; n=23 ; p<0.001)$.

Cholesterol, HDL cholesterol and triglycerides in serum were measured with commercial assays (Boehringer Mannheim, Mannheim, Germany). The albumin concentration in urine was measured with radioimmunoassay.

Statistical methods. The values are presented as means \pm SD or median and range. The significance of differences between group means was tested with the Mann-Whitney U-test. Correlations were tested using the Spearman rank correlation coefficients. The relationships between dependent variables such as retinopathy, neuropathy and microalbuminuria and independent variables were tested with multiple logistic regression analysis using an SPSS program for Windows. A prerequisite was that there were at least five affected subjects in the groups. A $p$-value less than 0.05 was considered statistically significant.

\section{Results}

There were no significant differences in age, duration of diabetes, BMI, WHR, serum total and HDL cholesterol concentrations between MODY3 and IDDM patients (Table 1), but the MODY3 patients signs of neuropathy (see methods)

a Above mean +2 SD of an age-adjusted control population had higher triglyceride concentrations than the IDDM patients $(p<0.001)$. The MODY3 patients were younger $(p<0.001)$, had a lower BMI $(p<$ $0.001)$, lower total $(p<0.01)$ but higher HDL $(p<$ $0.01)$ cholesterol concentrations than the NIDDM patients. Twenty-one (37\%) MODY3 patients, all IDDM patients $(100 \%)$ but only $13(8.2 \%)$ NIDDM patients were treated with insulin.

Neuropathy: Four out of 38 patients $(11 \%)$ reported mild, $7 \%$ reported moderate and $5 \%$ reported severe neuropathic symptoms. Nine percent had mild, $5 \%$ had moderate and $5 \%$ had severe clinical sensory polyneuropathy. Taken together, 11 patients $(29 \%)$ could be considered to have clinical neuropathy. In two patients distal neuropathy had been diagnosed earlier by neurophysiological examination. In addition, the age-adjusted vibration threshold was elevated in $40 \%$ of the MODY3 patients, and all patients with clinical neuropathy had an elevated vibration threshold. In a multiple logistic regression analysis with elevated age-adjusted vibration threshold as dependent variable $\mathrm{HbA}_{1 \mathrm{c}}(p=0.03)$ and male sex $(p=0.04)$ were independent predictors of neuropathy, although the confidence intervals were large. However, if clinical neuropathy was used as dependent variable, only duration had a weak influence on the development of neuropathy.

Microalbuminuria: Nine (19\%) of the 48 patients with data on albumin excretion had AER more than $20 \mu \mathrm{g} / \mathrm{min}$, whereas $12(25 \%)$ had AER more than $15 \mu \mathrm{g} / \mathrm{min}$. In a multiple logistic regression analysis with AER greater than $15 \mu \mathrm{g} / \mathrm{min}$ as dependent variable and age, duration of diabetes, hypertension, male sex and clinical neuropathy as dependent variables, only $\mathrm{HbA}_{1 \mathrm{c}}$ was an independent predictor of an elevated AER $(p=0.04)$.

Retinopathy: Twenty of the MODY3 patients $(53 \%)$ had no signs of retinopathy, $13(34 \%)$ had mild non-proliferative diabetic retinopathy (NPDR), while 5 patients $(13 \%)$ had sever NPDR or prolifera- 
Table 5. Multiple logistic regression analysis with retinopathy as dependent variable

\begin{tabular}{llll}
\hline & Risk ratio & $\begin{array}{l}95 \% \text { Confidence } \\
\text { interval }\end{array}$ & $p$ value \\
\hline $\begin{array}{l}\text { Age (years) } \\
\text { Duration of diabetes } \\
\text { (years) }\end{array}$ & 0.99 & $0.92-1.07$ & 0.97 \\
HbA $_{1 \mathrm{c}}$ (\%) & 3.1 & $1.02-1.25$ & 0.03 \\
$\begin{array}{l}\text { Hypertension } \\
\text { Microalbuminuria }\end{array}$ & 1.7 & $1.1-8.6$ & 0.03 \\
\hline
\end{tabular}

Complete data for the independent variables were available on 36 patients, 14 of whom were considered to have retinopathy

tive retinopathy (PDR) (Table 2). There was, however, no significant difference in the prevalence of retinopathy between MODY3 and IDDM or NIDDM patients matched for duration and glycaemic control.

In the multiple logistic regression analysis with any retinopathy as dependent variable and age, duration of diabetes, $\mathrm{HbA}_{1 \mathrm{c}}$, hypertension and microalbuminuria as independent variables, $\mathrm{HbA}_{1 \mathrm{c}}$ was an independent predictor of retinopathy with a risk ratio of $3.1(p=0.03)$. Duration of diabetes also showed a weak association with retinopathy (RR 1.13, $p=$ $0.04)$.

Hypertension and macrovascular disease: The prevalence of hypertension was similar in MODY3 and IDDM (Table 1), but lower than in NIDDM $(p<0.001)$. Coronary heart disease was more often seen in MODY 3 than in IDDM patients (16 vs $4.5 \%$, $p<0.02$ ), but less frequently than in the older NIDDM patients (16 vs $33.3 \%, p<0.02)$. One asymptomatic patient had ECG findings indicating an old myocardial infarction, and one patient had undergone coronary bypass surgery. One patient had a history of ischaemic stroke with a persisting defect in the visual fields. None of the patients had signs of peripheral vascular disease, i.e. the ankle-brachial index was higher than 0.9 in all patients (mean $1.1 \pm 0.1$ ).

\section{Discussion}

The main findings of the current study were that microangiopathic and neuropathic complications were as common on MODY3 patients as in IDDM and NIDDM patients matched for duration and glycaemic control and that these complications were clearly determined by the degree of glycaemic control. In addition, coronary heart disease was more frequent in MODY3 than in IDDM patients despite similar prevalence of hypertension.

It is almost impossible to create a diabetic reference group which is matched for age, duration of diabetes and glycaemic control. We have therefore chosen to match the groups for duration of diabetes and glycaemic control, but not for age. This may partially explain the higher frequency of hypertension and coronary heart disease in the NIDDM than in the MODY3 or IDDM patients.

The findings support and extend a previous study from France [17] by providing standardised and controlled examinations of retinopathy, neuropathy, albuminuria and macrovascular disease in the MODY3 patients. In the French study the presence of complications was based upon information from local hospital records in 45 affected MODY3 patients from 7 kindreds [17]. In the current study, the prevalence of mild $(34,37$ and $21 \%)$ and severe non-proliferative or proliferative $(13,16$ and $18 \%)$ retinopathy was similar in the MODY3, IDDM and the NIDDM patients matched for duration of diabetes and $\mathrm{HbA}_{1 \mathrm{c}}$. In the French study, the prevalence of proliferative retinopathy was $21 \%$ in the MODY3 patients, which was similar to the prevalence in the NIDDM patients $(23 \%)$. In this study, the level of glycaemic control was a strong determinant of retinopathy, with an increase in the risk ratio by 3.1 for each percent increase in $\mathrm{HbA}_{1 \mathrm{c}}$. Although this figure is higher than that observed in Swedish IDDM patients $(1.37$ with a $95 \%$ confidence interval of 1.14 to 1.64 [18), it should be kept in mind that the confidence interval is wide (1.1 to 8.6) due to the small number of subjects studied. It, however, seems to be higher than the risk ratio of 1.08 (95\% CI 1.0-1.17) which we have reported in Swedish NIDDM patients [18]. Glycaemic control was also associated with retinopathy in the French study [17]. However, the risk seemed to be lower than in NIDDM patients as indicated by an odds ratio of 2.69 for the development of retinopathy in NIDDM compared with MODY3 patients. This odds ratio was, however, much lower in MODY2 compared with MODY3 patients (0.27).

Taken together the data clearly indicate that severe retinopathy occurs in MODY3 patients and that these eye complications are determined by the degree of glycaemic control, and to a lesser extent by the duration of the disease.

The prevalence of microalbuminuria defined as overnight urine excretion more than $20 \mu \mathrm{g} / \mathrm{min}$ (or $15 \mu \mathrm{g} / \mathrm{min}$ ) did not differ between MODY3 and IDDM or NIDDM patients. Four MODY3 patients had macroalbuminuria and one of them was treated for uraemia. The prevalence of microalbuminuria also seems to be similar to that reported in IDDM patients with a long duration of diabetes [19]. In addition, the prevalence of microalbuminuria in patients with newly diagnosed NIDDM is about $20 \%$ and the prevalence does not increase much during the following 5 years [20] in contrast to the situation in IDDM, in NIDDM microalbuminuria seems to reflect widespread vascular damage rather than incipient nephropathy, and albuminuria is a strong predictor of cardiovascular death. In NIDDM, microalbuminuria is usually associated with hypertension, dyslipidaemia 
and coronary heart disease, i.e. components of the metabolic syndrome. In the MODY3 patients $\mathrm{HbA}_{1 \mathrm{c}}$, but neither the duration of the disease nor hypertension predicted the presence of microalbuminuria. In patients with IDDM hypertension in addition to $\mathrm{HbA}_{1 \mathrm{c}}$ predicts subsequent microalbuminuria (B. Isomaa, unpublished observations). Although we do not know whether microalbuminuria in MODY3 reflects incipient nephropathy or endothelial dysfunction, the lack of other components of the metabolic syndrome in MODY3 supports the former view. Also the French study found a trend towards a higher prevalence of nephropathy defined as dipstick-positive proteinuria in MODY3 patients compared with older NIDDM patients of shorter duration (19 vs $7 \%$ ) [17].

These data strongly support the view that MODY3 patients are susceptible to develop nephropathy as a consequence of elevated blood glucose levels and that the severity of glycaemia is more important for the development of diabetic nephropathy than the duration of the disease and the presence of hypertension.

The wide variation in the reported prevalence of peripheral diabetic neuropathy can most likely be explained by different diagnostic criteria and methods employed in addition to the inclusion of heterogeneous groups of diabetic patients. In this study we examined a homogenous subgroup of genetically defined diabetic patients by employing standardised methods for the assessment of sensory neuropathy.

Using the minimum criteria for symptomatic or clinical neuropathy (see methods) we found clinical neuropathy in $24 \%$ of our MODY 3 patients, $40 \%$ of whom had an elevated vibration threshold as assessed by biothesiometer. The discrepancy between the two measurements was greater in the IDDM patients, $41 \%$ of whom had elevated vibration threshold while only $13 \%$ had a positive clinical score. For comparison, in the UK Hospital Clinic Study [11] similar diagnostic criteria to those used in this study gave a neuropathy prevalence of $22.7 \%$ in IDDM patients with a median age of 45 years and a disease duration of 13 years. The corresponding figure in NIDDM patients was $32.1 \%$. Unfortunately we did not have the opportunity to study the NIDDM patients using the same standardised methodology. In another study, the prevalence of sensory neuropathy in IDDM was $17.1 \%$ (median age 33 years, duration of diabetes 10.8 years, $\mathrm{HbA}_{1 \mathrm{c}} 8.5 \%$ ) and in NIDDM $34.8 \%$ (median age 54 years, duration of diabetes 9 years and $\mathrm{HbA}_{1 \mathrm{c}} 8 \%$ ) [21]. If we used the vibration threshold measured by the biothesiometer as the dependent variable and duration of diabetes and $\mathrm{HbA}_{1 \mathrm{c}}$ as independent variables, only $\mathrm{HbA}_{1 \mathrm{c}}$ predicted sensory neuropathy. If we used the clinical score as dependent variable, only duration of diabetes was weakly associated with neuropathy, and this did not change if we excluded age, hypertension and male sex from the analy- sis. We think that the most likely explanation for this discrepancy is that vibration threshold represents a more standardised and sensitive indicator of distal sensory neuropathy than the clinical score.

We observed similar prevalences of hypertension in the MODY3 and the IDDM patients (25 and $19 \%)$. These figures were lower than the figures in the older NIDDM patients (54\%).

There was no relationship between hypertension and $\mathrm{HbA}_{1 \mathrm{c}}$ or the duration of diabetes in this study, suggesting that other mechanisms than those contributing to the development of microangiopathy and neuropathy are operative for hypertension. Furthermore, coronary heart disease was seen in $16 \%$ of the MODY3 patients, which was significantly higher than in the IDDM patients $(4.5 \%)$ but lower than in the NIDDM patients ( $33 \%$ ). This figure should also be contrasted against a prevalence of $53 \%$ in newly diagnosed Finnish male and $40 \%$ in Finnish female NIDDM patients aged less than 50 years [22] using similar diagnostic criteria.

Both hypertension and coronary heart disease have been associated with insulin resistance [23]. One explanation for the lower frequency of hypertension and coronary heart disease in MODY3 than in NIDDM could be that MODY3 patients show normal or even increased insulin sensitivity [9].

In conclusion, microangiopathic complications including neuropathy occur with the same frequency in MODY3 as in IDDM and NIDDM and hyperglycaemia is the main predictor of these complications. Hypertension and macrovascular disease is less frequent in MODY3 than in NIDDM; one possible explanation could be that MODY3 patients do not exhibit the insulin resistance characteristic of NIDDM. Since MODY3 patients are very sensitive to small increments in blood glucose levels and the disease can be undiagnosed for years [9], screening for persons at risk and early treatment should be recommended.

Acknowledgements. The authors are grateful to Eva Steffert for grading of photographs, to the Clinical Chemistry Laboratory of the Karleby Central Hospital for $\mathrm{HbA}_{1 \mathrm{c}}$ measurements, to Topcon Optical Svenska AB for supplying us with a Topcon NW5 camera for fundus photography, and to the Botnia research group and the staff of Perhonjokilaakso Health Center for technical assistance and support. The study was supported by grants from the Sigrid Juselius Foundation (L.G.), EEC (L.G.), Arvid Ohlssons Foundation (M.H.), Finnish Medical Society (Finska läkaresällskapet) (B.I., C.F.), the Lehikoinen Foundation (B. I.) and the Albert Påhlsson Foundation (M. L.).

\section{References}

1. Tattersall R (1973) Mild familial diabetes with dominant inheritance. Quart J Medicine 43: 339-357

2. Fajans S, Floyd Jr J, Tattersall R, Williamsson J, Pek S, Taylor C (1976) The various faces of diabetes in the young. Arch Intern Med 136: 194-202 
3. Yamagata K, Furuta H, Oda N et al. (1996) Mutations in the hepatocyte nuclear factor- $4 \alpha$ in maturity-onset diabetes of the young (MODY1). Nature 384: 458-460

4. Froguel P, Vaxillaire M, Sun F et al. (1992) Close linkage of glucokinase locus on chromosome $7 \mathrm{p}$ to early-onset noninsulin-dependent diabetes mellitus. Nature 356: 162-167

5. Yamagata K, Oda N, Kaisaki PJ et al. (1996) Mutations in the hepatocyte nuclear factor- $1 \alpha$ gene in maturity-onset diabetes of the young (MODY3). Nature 384: 455-458

6. Vaxillaire M, Boccio V, Philippi A et al. (1995) A gene for early-onset non-insulin-dependent diabetes mellitus (maturity-onset diabetes of the young) maps to chromosome 12q. Nature Genetics 9: 418-423

7. Velho G, Froguel P, Clèment K et al. (1992) Primary pancreatic beta-cell secretory defect caused by mutations in glucokinase gene in kindreds of maturity onset diabetes of the young. Lancet 340: 444-448

8. Glucksmann SA, Lehto M, Tayber O et al. (1997) Novel mutations and a mutational hotspot in the MODY3 gene. Diabetes 46: 1081-1086

9. Lehto M, Tuomi T, Mahtani M et al. (1997) Early-onset diabetes linked to chromosome 12 (MODY3) is characterized by a severe insulin secretion defect. J Clin Invest 99: 582-591

10. Groop L, Forsblom C, Lehtovirta M et al. (1996) Metabolic consequences of a family history of NIDDM: The Botnia Study. Evidence for sex-specific parental effects. Diabetes 45: $1585-1593$

11. Young M, Boulton A, Macleod A, Williams D, Sonksen P (1993) A multicentre study of the prevalence of diabetic peripheral neuropathy in the United Kingdom hospital clinic population. Diabetologia 36: $150-154$

12. Forsblom C, Eriksson J, Ekstrand A, Teppo A-M, Taskinen M-R, Groop L (1995) Insulin resistance and abnormal albumin excretion in non-diabetic first-degree relatives of patients with NIDDM. Diabetologia 38: 363-369

13. Klein R, Klein BEK, Moss SE, Davis MD, DeMets DL (1989) The Wisconsin Epidemiologic Study of Diabetic Re- tinopathy IX. Four-year incidence and progression of diabetic retinopathy when age at diagnosis is less than 30 years. Arch Ophthalmol 107: 237-243

14. Klein R, Klein B, Magli Y, Brothers R, Meuer S, Davis M (1986) An alternative method of grading diabetic retinopathy. Ophthalmology 93: 1183-1187

15. Rose G, Blackburn H (1968) Cardiovascular survey methods. WHO Monograph Series. Vol.56. WHO, Geneva

16. Jeppsson JO, Jerntorp P, Sundqvist G, Englund H, Nylund V (1986) Measurement of hemoglobin A1c by a new liquid-chromatographic assay: Methodology, clinical utility, and relation to glucose tolerance evaluated. Clin Chem 32: $1867-1872$

17. Velho G, Vaxillaire M, Boccio V, Charpentier G, Froguel P (1996) Diabetic complications in NIDDM kindreds linked to the MODY3 locus on chromosome 12q. Diabetes Care 19: 915-919

18. Henricsson M, Nilsson A, Groop L, Heijl A (1996) Prevalence of diabetic retinopathy in relation to age at onset of the diabetes, treatment, duration and glycemic control. Acta Ophthalmol Scand 74: 523-527

19. Forsblom C, Groop P-H, Groop L (1992) Low predictive value of microalbuminuria in patients with insulin-dependent diabetes of long duration. Brit Med J 305: 1051-1053

20. Niskanen L, Uusitupa M, Sarlund H, et al. (1990) Microalbuminuria predicts the development of serum lipoprotein abnormalities favouring atherogenesis in newly diagnosed type 2 diabetic patients. Diabetologia 33: 237-243

21. Ziegler D, Gries F, Muhlen H, Rathman W, Spuler M, Lessman F and the DIACAN multicenter study group (1993) Prevalence and clinical correlates of cardiovascular autonomic and peripheral diabetic neuropathy in patients attending diabetes centers. Diabete Metab 19: 143-151

22. Uusitupa M (1983) Coronary heart disease and left ventricular performance in newly diagnosed non-insulin-dependent diabetics. Kuopio University, Finland, pp 152

23. Reaven G (1988) Role of insulin resistance in human disease. Diabetes 37: 1595-1607 